\title{
Component-Based Construction of Deadlock-Free Systems
}

\author{
Extended Abstract \\ Gregor Gössler ${ }^{1}$ and Joseph Sifakis ${ }^{2}$ \\ 1 INRIA Rhône-Alpes, goessler@inrialpes.fr \\ 2 VERIMAG, sifakis@imag.fr
}

\begin{abstract}
We propose a framework for building deadlock-free systems from deadlock-free components. The framework is based on a methodology for the layered construction of systems by superposing three layers. A layer of components, an interaction model and a restriction layer. The interaction model specifies the possible interactions between components. The restriction layer restricts the behavior of the two lower layers by a global constraint. Layered structuring allows separating three orthogonal aspects in system construction. Apart from its methodological interest it makes technically possible the definition of a unique and powerful associative composition operator.

We study sufficient deadlock-freedom conditions for systems built from deadlock-free components and given interaction model and restriction. We also provide a sufficient condition for individual deadlock-freedom of the components of such systems.
\end{abstract}

\section{Introduction}

Deadlock-freedom is an essential correctness property as it characterizes a system's ability to perform some activity over its lifetime. Deadlocks are the most common source of errors in systems of concurrent processes. They occur when processes share common resources or are in general subject to strong synchronization constraints. In that case, a process may remain blocked as long as a condition depending on the state of their environment is not satisfied.

It has been often argued that deadlock-freedom is not a relevant property as there exist systems that never deadlock e.g. hardware, time triggered systems $[12,10]$, synchronous systems $[4,9,2,14]$. In such systems, components are never blocked by their environment as it is supposed that component inputs are always available whenever requested. Nevertheless, it is clear that if some strong coordination between components is needed e.g. they share a common resource, then this can be achieved by a deadlock-free protocol where the values exchanged between components are used to encode the information necessary for safely implementing the coordination. Thus, for systems that are by construction deadlock-free, verifying mutual exclusion requires analysis of the sequences of the values exchanged between the coordinating components. 
Another argument for not considering deadlock-freedom as a relevant property is that any system can become trivially deadlock-free by adding some idle action loop that does not modify its overall observable behavior whenever there is a risk of deadlock e.g. at waiting states. Such a modification allows elimination of deadlocks but it leads to systems where it is possible to indefinitely postpone interaction between components by privileging idle actions. Thus instead of checking for deadlock-freedom, other properties such as livelock-freedom and fairness must be checked for system correctness.

The above remarks show that building deadlock-free systems requires the definition of an appropriate setting where absence of deadlock means satisfaction of strong coordination properties. In this paper we propose a framework for building deadlock-free systems from deadlock-free components. The framework is based on a methodology for the layered construction of systems by superposing three layers (figure 1).

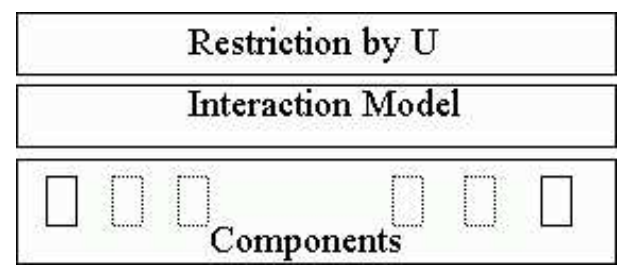

Fig. 1: Layered system description.

- The bottom layer consists of a set of components. They are characterized by the set of actions they can perform and their behavior. The latter is specified as a transition system representing the effect of actions on component states.

- The intermediate layer is an interaction model. This is used to specify the possible interactions between the components. An interaction is the result of the simultaneous occurrence (synchronization) of actions from different components. Furthermore, an interaction model specifies the set of incomplete interactions, that is the interactions that the system cannot execute without synchronizing with its environment. Interaction models are a general mechanism for specifying various kinds of parallel composition [8].

- The upper layer is a restriction by a constraint (predicate) on the system state. Restriction is used to prevent the behavior of the underlying layers from executing interactions that violate the constraint.

System behavior is obtained by successive application of the meaning of each layer. The behavior of components is composed as specified by the interaction model. Then it is restricted by application of a global constraint. We believe that the proposed construction methodology is general enough as it combines 
usual parallel composition with techniques relying on the use of constraints such as invariants. For example, in a multitasking system, interaction models can be typically used to describe synchronization for mutual exclusion and restriction can be used to express scheduling policies [1].

We provide sufficient conditions for deadlock-freedom of systems built from deadlock-free components for given interaction model and restriction. The results assume that for each component a deadlock-free invariant is given, that is a set of states from which the component can block only because of its environment. The condition relates the deadlock-free invariants of the system components to the enabling conditions of the interactions of the layered system.

We also provide a sufficient condition for individual deadlock-freedom of the components in a system. A component is individually deadlock-free if it can always perform an action.

The paper is organized as follows.

Section 2 describes the layered construction principle. It deals essentially with the presentation of the interaction models and their properties. Component behaviors are described as simple non deterministic loops with guarded commands $[7,16]$. The concept of restriction is taken from [1].

Section 3 presents the result for global and individual deadlock-freedom. Section 4 presents concluding remarks about the presented framework.

\section{Composition}

We present a composition operation on layered systems. The operation composes each layer separately. The bottom layer of the product is the union of the bottom layers of the operands. The interaction model of the product is obtained as the union of the interaction models of the operands with some "glue" interaction model. That is, the composition operator is parameterised with information about the interactions between the operands. Finally, the restriction of the product is the conjunction of the restrictions of the operands (figure 2).

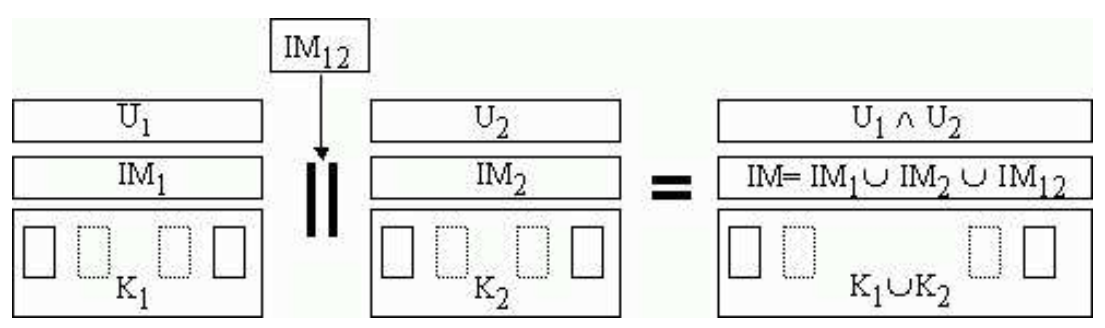

Fig. 2: Layered composition.

The two following sub-sections present the interaction models and their composition. 


\subsection{Interaction Models}

Consider a finite set $K$ of components with disjoint finite vocabularies of actions $A_{i}$ for $i \in K$. We put $A=\bigcup_{i \in K} A_{i}$.

A connector $c$ is a non empty subset of $A$ such that $\forall i \in K .\left|A_{i} \cap c\right| \leqslant 1$. A connector defines a maximally compatible set of interacting actions. For the sake of generality, our definition accepts singleton connectors. The use of the connector $\{a\}$ in a description is interpreted as the fact that action $a$ cannot be involved in interactions with other actions.

Given a connector $c$, an interaction $\alpha$ of $c$ is any term of the form $\alpha=a_{1}$ । $\ldots \mid a_{n}$ such that $\left\{a_{1}, \ldots, a_{n}\right\} \subseteq c$. As usual [15,3], we assume that । is a binary associative and commutative operator. It is used to denote some abstract and partial action composition operation. The interaction $a_{1}|\ldots| a_{n}$ is the result of the occurrence of the actions $a_{1}, \ldots, a_{n}$. For $\alpha$ and $\alpha^{\prime}$ interactions, we write $\alpha$ । $\alpha^{\prime}$ to denote the interaction resulting from their composition (if its is defined).

Notice that if $\alpha=a_{1}|\ldots| a_{n}$ is an interaction then any term corresponding to a sub-set of $\left\{a_{1}, \ldots, a_{n}\right\}$ is an interaction. By analogy, we say that $\alpha^{\prime}$ is a sub-interaction of $\alpha$ if $\alpha=\alpha^{\prime}$ । $\alpha^{\prime \prime}$ for some interaction $\alpha^{\prime \prime}$. Clearly,actions are minimal interactions.

The set of the interactions of a connector $c=\left\{a_{1}, \ldots, a_{n}\right\}$, denoted by $I(c)$, consists of all the interactions corresponding to sub-sets of $c$ (all the subinteractions of $c$ ). We extend the notation to sets of connectors. If $C$ is a set of connectors then $I(C)$ is the set of its interactions. Clearly, for $C_{1}, C_{2}$ sets of connectors, $I\left(C_{1} \cup C_{2}\right)=I\left(C_{1}\right) \cup I\left(C_{2}\right)$.

Definition 1 (Set of connectors). The set of connectors of a system consisting of a set of components $K$ with disjoint action vocabularies $A_{i}$ for $i \in K$, is a set $C$ such that $\bigcup_{c \in C} c=\bigcup_{i \in K} A_{i}$, and if $c \in C$ then there exists no $c^{\prime} \in C$ and $c \subsetneq c^{\prime}$. That is, $C$ contains only maximal sets.

Definition 2 (Interaction model). The interaction model of a system composed of a set of components $K$ with a set of connectors $C$ is a pair $I M=$ $\left(I(C), I(C)^{-}\right)$where $I(C)^{-} \subseteq I(C)$, is the set of the incomplete interactions such that it contains no maximal interactions and $\forall b, b^{\prime} \in I(C), b \in I(C)^{-}$and $b^{\prime} \subseteq b$ implies $b^{\prime} \in I(C)^{-}$. We denote by $I(C)^{+}$the set of the complete (non incomplete) interactions. Clearly, $I(C)^{+}$contains all the maximal interactions of $I(C)$ and is such that $\forall b, b^{\prime} \in I(C), b \in I(C)^{+}$and $b \subseteq b^{\prime}$ implies $b^{\prime} \in I(C)^{+}$.

Notice that any action appears in some connector. The requirement that $C$ contains only maximal sets ensures a bijective correspondence between the set of connectors $C$ and the corresponding set of interactions $I(C)$. Given $I(C)$, the corresponding set of connectors is uniquely defined and is $C$. To simplify notation, we write $I C$ instead of $I(C)$.

The distinction between complete and incomplete interactions is essential for building correct models. As models are built incrementally, interactions are obtained by composing actions. It is often necessary to express the constraint that some interactions of a sub-system are not interactions of the system. This is 
typically the case for binary strict synchronization (rendez-vous). For example, send and receive should be considered as incomplete actions but sendıreceive as complete. The occurrence of send or receive alone in a system model is an error because it violates the assumption about strict synchronization made by the designer.

The execution of a complete interaction by a component does not require synchronization with interactions of its environment. The execution of an incomplete interaction requires synchronization with some other interaction to produce a larger one which may be either complete or incomplete. Incompleteness of an interaction implies the obligation to synchronize when environment offers matching interactions as specified by the connectors.

The distinction between complete and incomplete interactions encompasses many other distinctions such as output/input, internal/external, uncontrollable/ controllable used in different modeling formalisms. Clearly, internal actions of components should be considered as complete because they can be performed independently of the state of their environment. In some formalisms, output actions are complete (synchronous languages, asynchronous buffered communication). In some others such as CSP [11] and Lotos [17], all synchronizing actions are incomplete.

Often it is convenient to consider that the complete interactions of $I C^{+}$are defined from a given set of complete actions $A^{+} \subseteq A$. That is, $I C^{+}$consists of all the interactions of $I C$ where at least one complete action (element of $A^{+}$) is involved. In the example of figure 3, we give sets of connectors and complete actions to define interaction models. By convention, bullets represent incomplete actions and triangles complete actions. In the partially ordered set of the interactions, full nodes denote complete interactions. The interaction between put and get represented by the interaction putiget is a rendez-vous meaning that synchronization is blocking for both actions. The interaction between out and in is asymmetric as out can occur alone even if in is not possible. Nevertheless, the occurrence of in requires the occurrence of out. The interactions between out, $i n_{1}$ and $i n_{2}$ are asymmetric. The output out can occur alone or in synchronization with any of the inputs $i n_{1}, i n_{2}$.

\subsection{Incremental Description of Interaction Models}

Consider the interaction model $I M=\left(I C, I C^{-}\right)$of a set of interacting components $K$ with disjoint action vocabularies $A_{i}$ for $i \in K . I C$ and $I C^{-}$denote the sets of interactions and incomplete interactions, respectively on the vocabulary of actions $A=\bigcup_{i \in K} A_{i}$.

Definition 3 (Glue connectors). Given a set of disjoint subsets of $K, K_{1}, \ldots$, $K_{n}$ of $K$, we denote by $C\left[K_{1}, \ldots, K_{n}\right]$ the set of the connectors having at least one action in each set of components, that is, $C\left[K_{1}, \ldots, K_{n}\right]=\left\{c=c_{1} \cup \cdots \cup c_{n} \mid\right.$ $\left.\forall i \in[1, n] . c_{i} \in C\left[K_{i}\right] \wedge c \in C[K]\right\}$.

Clearly, $C\left[K_{1}, \ldots, K_{n}\right]$ is the set of the connectors of $I M\left[K_{1} \cup \cdots \cup K_{n}\right]$ which are not connectors of any $I M\left[K^{\prime}\right]$ for any subset $K^{\prime}$ of at most $n-1$ elements 


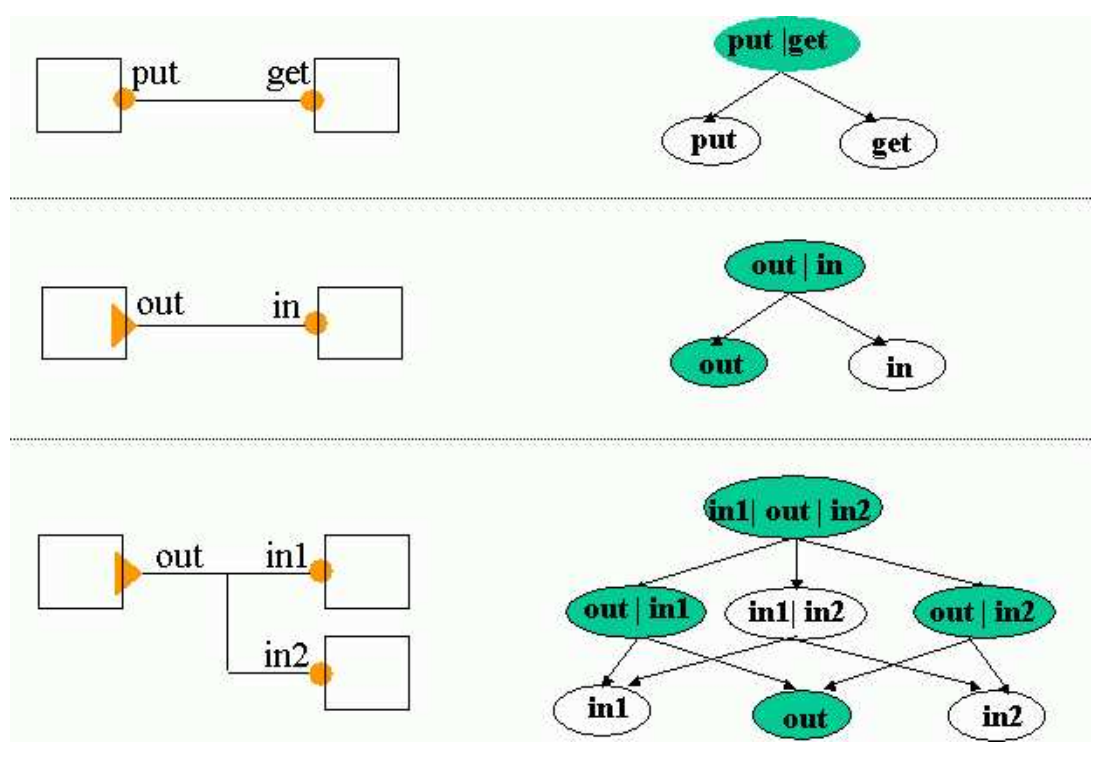

Fig. 3: Interaction models.

from $\left\{K_{1}, \ldots, K_{n}\right\}$. Notice that when the partition consists of only one set, then the above definition agrees with Definition 1.

Proposition 1. Given $K_{1}, K_{2}, K_{3}$ three disjoint subsets of $K$.

$$
\begin{aligned}
I C\left[K_{1} \cup K_{2}\right] & =I C\left[K_{1}\right] \cup I C\left[K_{2}\right] \cup I C\left[K_{1}, K_{2}\right] \\
I C\left[K_{1} \cup K_{2}, K_{3}\right] & =I C\left[K_{1}, K_{3}\right] \cup I C\left[K_{2}, K_{3}\right] \cup I C\left[K_{1}, K_{2}, K_{3}\right]
\end{aligned}
$$

Proof. The first equality comes from the fact that $C\left[K_{1}\right] \cup C\left[K_{2}\right] \cup C\left[K_{1}, K_{2}\right]$ contains all the connectors of $C\left[K_{1} \cup K_{2}\right]$ and other sets of actions that are not maximal. By definition, $I C$ contains all the sub-sets of $C$. Thus, $I C\left[K_{1} \cup K_{2}\right]=$ $I\left(C\left[K_{1}\right] \cup C\left[K_{2}\right] \cup C\left[K_{1}, K_{2}\right]\right)=I C\left[K_{1}\right] \cup I C\left[K_{2}\right] \cup I C\left[K_{1}, K_{2}\right]$.

The second equality comes from the fact that $C\left[K_{1}, K_{3}\right] \cup C\left[K_{2}, K_{3}\right] \cup C\left[K_{1}\right.$, $\left.K_{2}, K_{3}\right]$ contains all the connectors of $C\left[K_{1} \cup K_{2}, K_{3}\right]$ and in addition, other sets of actions that are not maximal. By definition, $I C$ contains all the sub-sets of $C$. Thus, $I C\left[K_{1} \cup K_{2}, K_{3}\right]=I\left(C\left[K_{1}, K_{3}\right] \cup C\left[K_{2}, K_{3}\right] \cup C\left[K_{1}, K_{2}, K_{3}\right]\right)$ from which we get the result by distributivity of $I$ over union.

Definition 4 (Union of incomplete interactions). Consider two sets of connectors $C_{1}, C_{2}$ and the corresponding sets of interactions $I C_{1}, I C_{2}$. We take $\left(I C_{1}\right)^{-} \cup\left(I C_{2}\right)^{-}=\left(I C_{1} \cup I C_{2}\right)^{-}$.

This definition combined with proposition 1 allows to compute the incomplete interactions of a system from the incomplete interactions of its components and thus provides a basis for incremental description of interaction models. 
Property 1. For $K_{1}, K_{2}, K_{3}$ three disjoint subsets of $K$,

$$
\begin{aligned}
I C\left[K_{1} \cup K_{2}\right]^{-} & =I C\left[K_{1}\right]^{-} \cup I C\left[K_{2}\right]^{-} \cup I C\left[K_{1}, K_{2}\right]^{-} \\
I M\left[K_{1} \cup K_{2}\right] & =\left(I C\left[K_{1} \cup K_{2}\right], I C\left[K_{1} \cup K_{2}\right]^{-}\right) \\
& =I M\left[K_{1}\right] \cup I M\left[K_{2}\right] \cup I M\left[K_{1}, K_{2}\right] \\
I M\left[K_{1} \cup K_{2}, K_{3}\right] & =I M\left[K_{1}, K_{3}\right] \cup I M\left[K_{2}, K_{3}\right] \cup I M\left[K_{1}, K_{2}, K_{3}\right]
\end{aligned}
$$

By using this property, we get the following expansion formula:

\section{Proposition 2 (Expansion formula).}

$$
\begin{aligned}
I M\left[K_{1} \cup K_{2} \cup K_{3}\right]= & I M\left[K_{1}\right] \cup I M\left[K_{2}\right] \cup I M\left[K_{3}\right] \cup I M\left[K_{1}, K_{2}\right] \\
& \cup I M\left[K_{1}, K_{3}\right] \cup I M\left[K_{2}, K_{3}\right] \cup I M\left[K_{1}, K_{2}, K_{3}\right] .
\end{aligned}
$$

\subsection{Composition Semantics and Properties}

We need the following definitions.

Definition 5 (Transition system). A transition system $B$ is a tuple $(X, I C$, $\left.\left\{G^{a}\right\}_{a \in I C},\left\{F^{a}\right\}_{a \in I C}\right)$, where

- $X$ is a finite set of variables;

- IC is a finite set of interactions;

- $G^{a}$ is a predicate on $\mathbf{X}$, the set of valuations of $X$, called guard;

$-F^{a}: \mathbf{X} \rightarrow \mathbf{X}$ is a transition function.

Definition 6 (Semantics of a transition system). A transition system $(X$, $\left.I C,\left\{G^{a}\right\}_{a \in I C},\left\{F^{a}\right\}_{a \in I C}\right)$ defines a transition relation $\rightarrow: \mathbf{X} \times I C \times \mathbf{X}$ as follows: $\forall \mathbf{x}, \mathbf{x}^{\prime} \in \mathbf{X} \forall a \in I C . \mathbf{x} \stackrel{a}{\rightarrow} \mathbf{x}^{\prime} \Longleftrightarrow G^{a}(\mathbf{x}) \wedge \mathbf{x}^{\prime}=F^{a}(\mathbf{x})$.

Definition 7 (Constraint). A constraint on a transition system $B=(X, I C$, $\left.\left\{G^{a}\right\}_{a \in I C},\left\{F^{a}\right\}_{a \in I C}\right)$ is a term of the form $U^{X} \wedge \bigwedge_{a \in I C} t^{a}\left(U^{a}\right)$, where

- $U^{X}$ is a state predicate on $X$;

- $t^{a}\left(U^{a}\right)$ is an action predicate such that $\forall \mathbf{x} \in \mathbf{X} \cdot t^{a}\left(U^{a}\right)(\mathbf{x})$ if $G^{a}(\mathbf{x}) \Rightarrow$ $U^{a}(\mathbf{x})$.

That is, $U^{X} \wedge \bigwedge_{a \in I C} t^{a}\left(U^{a}\right)$ holds at states which satisfy $U^{X}$ and from which only actions satisfying $U^{a}$ are executable.

Definition 8 (Restriction). The restriction of a transition system $B=(X$, $\left.I C,\left\{\left(G^{a}\right)\right\}_{a \in I C},\left\{F^{a}\right\}_{a \in I C}\right)$ with a constraint $U=U^{X} \wedge \bigwedge_{a \in I C} t^{a}\left(U^{a}\right)$ is the transition system $B / U=\left(X, I C,\left\{\left(G^{a}\right)^{\prime}\right\}_{a \in I C},\left\{F^{a}\right\}_{a \in I C}\right)$ where $\left(G^{a}\right)^{\prime}=G^{a} \wedge U^{X} \wedge$ $U^{a} \wedge U^{X}\left(\left[F^{a}(\mathbf{x}) / \mathbf{x}\right]\right)$. 
A system $S$ is described by a term $S=(B, I M) / U$ where $I M=\left(I C, I C^{-}\right)$ is an interaction model, $B$ is a transition system with set of interactions $I C$ describing the behavior of its components, and $U$ is a constraint.

We define the behavior of $S$ as the transition system $B^{\prime} / U$ where $B^{\prime}$ is the transition system obtained from $B$ by removing all its incomplete interactions, that is $B^{\prime}=\left(X, I C,\left\{G^{a}\right\}_{a \in I C^{+}},\left\{F^{a}\right\}_{a \in I C^{+}}\right)$, where $I C^{+}$is the set of the complete interactions of $I M$.

As in the previous sub-section, we consider that $S=(B, I M) / U$ is built from a set of interacting components $K$ with disjoint action vocabularies $A_{i}, i \in$ $K$ and behaviors described by transition systems $\left(X_{i}, A_{i},\left\{G^{a}\right\}_{a \in A_{i}},\left\{F^{a}\right\}_{a \in A_{i}}\right)$ with disjoint sets of variables.

We denote by $S[K]$ the system built from the components $k \in K$, and assume that it has the layered structure $S[K]=(B[K], I M[K]) / U$ with interaction model $I M[K]$ and constraint $U$.

We define a composition operator $\|$ allowing to obtain for disjoint sub-sets $K_{1}, K_{2}$ of $K$, the system $S\left[K_{1} \cup K_{2}\right]$ as the composition of the sub-systems $S\left[K_{1}\right]$, $S\left[K_{2}\right]$ for given interaction model $I M\left[K_{1}, K_{2}\right]$ connecting the two sub-systems. The operator composes separately behaviors, interaction models and restrictions of the sub-systems.
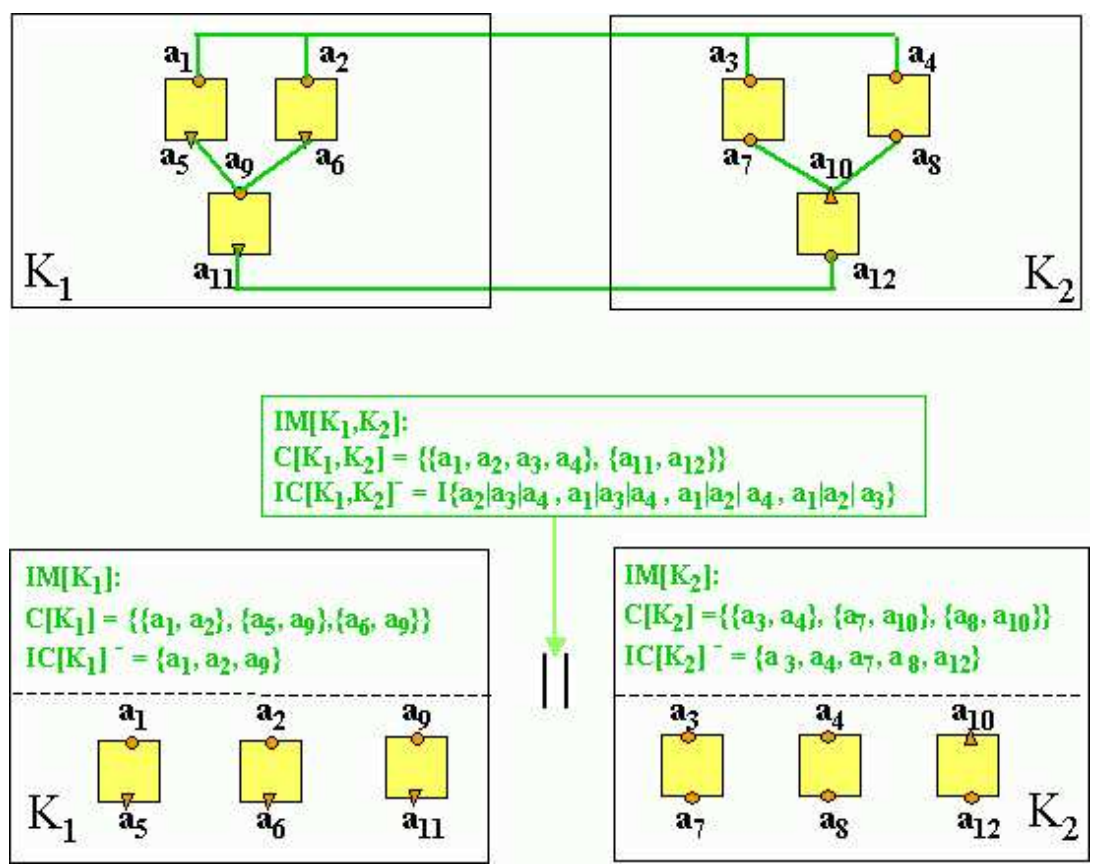

Fig. 4: The composition principle. 
Definition $9(\|)$. The composition of two systems $S\left[K_{1}\right]$ and $S\left[K_{2}\right]$ is the system

$$
\begin{aligned}
S\left[K_{1} \cup K_{2}\right] & =\left(B\left[K_{1}\right], I M\left[K_{1}\right]\right) / U_{1} \|\left(B\left[K_{2}\right], I M\left[K_{2}\right]\right) / U_{2} \\
& =\left(B\left[K_{1}\right] \times B\left[K_{2}\right], I M\left[K_{1}\right] \cup I M\left[K_{2}\right] \cup I M\left[K_{1}, K_{2}\right]\right) /\left(U_{1} \wedge U_{2}\right)
\end{aligned}
$$

where $\times$ is a binary associative behavior composition operator such that $B\left[K_{1}\right] \times$ $B\left[K_{2}\right]=\left(X_{1} \cup X_{2}, I C\left[K_{1} \cup K_{2}\right],\left\{G^{a}\right\}_{a \in I C\left[K_{1} \cup K_{2}\right]},\left\{F^{a}\right\}_{a \in I C\left[K_{1} \cup K_{2}\right]}\right)$ where for $\alpha=\alpha_{1} \mid \alpha_{2} \in I C\left[K_{1}, K_{2}\right], G^{\alpha}=G^{\alpha_{1}} \wedge G^{\alpha_{2}}$ and for any valuation $\left(\mathbf{x}_{1}, \mathbf{x}_{2}\right)$ of $X_{1} \cup X_{2}, F^{\alpha}\left(\mathbf{x}_{1}, \mathbf{x}_{2}\right)=\left(F^{\alpha_{1}}\left(\mathbf{x}_{1}\right), F^{\alpha_{2}}\left(\mathbf{x}_{2}\right)\right)$.

Due to property 1 we have $\left(B\left[K_{1}\right], I M\left[K_{1}\right]\right) / U_{1} \|\left(B\left[K_{2}\right], I M\left[K_{2}\right]\right) / U_{2}=$ $\left(B\left[K_{1} \cup K_{2}\right], I M\left[K_{1} \cup K_{2}\right]\right) /\left(U_{1} \wedge U_{2}\right)$, which means that composition of subsystems gives the system corresponding to the union of their components.

Notice that as $\times$ is an associative operator, composition is associative:

$$
\begin{aligned}
( & \left.\left(B\left[K_{1}\right], I M\left[K_{1}\right]\right) / U_{1} \|\left(B\left[K_{2}\right], I M\left[K_{2}\right]\right) / U_{2}\right) \|\left(B\left[K_{3}\right], I M\left[K_{3}\right]\right) / U_{3}= \\
= & \left(B\left[K_{1} \cup K_{2}\right], I M\left[K_{1} \cup K_{2}\right]\right) /\left(U_{1} \wedge U_{2}\right) \|\left(B\left[K_{3}\right], I M\left[K_{3}\right]\right) / U_{3} \\
= & \left(B\left[K_{1}\right] \times B\left[K_{2}\right] \times B\left[K_{3}\right], I M\left[K_{1} \cup K_{2}\right] \cup I M\left[K_{3}\right] \cup I M\left[K_{1} \cup K_{2}, K_{3}\right]\right) / \\
& \left(U_{1} \wedge U_{2} \wedge U_{3}\right) \\
= & \left(B\left[K_{1} \cup K_{2} \cup K_{3}\right], I M\left[K_{1} \cup K_{2} \cup K_{3}\right]\right) /\left(U_{1} \wedge U_{2} \wedge U_{3}\right)
\end{aligned}
$$

by application of proposition 2 . Thus we have the proposition

Proposition 3. $\|$ is a commutative and associative operator on systems.

\section{Deadlock Freedom by Construction}

\subsection{Global Deadlock Freedom}

Definition 10 (Invariant). A constraint $U=U^{X} \wedge \bigwedge_{a \in I C} t^{a}\left(U^{a}\right)$ on $B=$ $\left(X, I C,\left\{G^{a}\right\}_{a \in I C},\left\{F^{a}\right\}_{a \in I C}\right)$ is an invariant if $\forall a \in I C \forall \mathbf{x} \in \mathbf{X} \cdot U^{X}(\mathbf{x}) \wedge$ $G^{a}(\mathbf{x}) \Rightarrow U^{X}\left(\left[F^{a}(\mathbf{x}) / \mathbf{x}\right]\right) \wedge U^{a}(\mathbf{x})$.

The following properties are easy to prove and relate restriction to invariants.

Property 2. Given a transition system $B$ and constraints $U, U_{1}, U_{2}$,

- $U$ is an invariant of $B / U$;

- if $U_{i}$ is an invariant of $B_{i}, i=1,2$, then $U_{1} \wedge U_{2}$ is an invariant of $\left(B_{1} \times B_{2}, I M\right)$ for any $I M$, and of $\left(B_{1} \times B_{2}, I M\right) / U$;

$-\left(B / U_{1}\right) / U_{2}=B /\left(U_{1} \wedge U_{2}\right)$

- if $U$ is an invariant of $B$ then $B / U$ is bisimilar to $B$ from any state satisfying $U$. 
As in the previous section, consider a system $S=\left(\times_{k \in K} B_{k}, I M\right) / U$ built from a set of interacting components $K$ where the transition systems $B_{k}=$ $\left(X_{k}, A_{k},\left\{G^{a}\right\}_{a \in A_{k}},\left\{F^{a}\right\}_{a \in A_{k}}\right)$ have disjoint action vocabularies and sets of variables. We assume that $I M=\left(I C, I C^{+}\right)$, and $B=\left(\times_{k \in K} B_{k}, I M\right)=\left(X, I C^{+}\right.$, $\left.\left\{G^{a}\right\}_{a \in I C^{+}},\left\{F^{a}\right\}_{a \in I C^{+}}\right)$. In this section we study deadlock-freedom of $S=B / U$ and of its components.

Let $B / U=\left(X, I C^{+},\left\{\left(G^{a}\right)^{\prime}\right\}_{a \in I C^{+}},\left\{F^{a}\right\}_{a \in I C^{+}}\right)$be the restriction of $B$ by some constraint $U=U^{X} \wedge \bigwedge_{a \in A} t^{a}\left(U^{a}\right)$ with restricted guards $\left(G^{a}\right)^{\prime}=G^{a} \wedge$ $U^{X} \wedge U^{a} \wedge U^{X}\left(\left[F^{a}(\mathbf{x}) / \mathbf{x}\right]\right)$ for any interaction $a \in I C^{+}$.

Definition 11 (Complete states). Given $S=(B, I M), U=U^{X} \wedge \bigwedge_{a \in A} t^{a}\left(U^{a}\right)$, and $k$ some component of $B$, the set of complete states of $k$, that is, states of $k$ from which its progression cannot be prevented by its environment, is characterized by the largest predicate complete $(k)$ on $X_{k}$ such that complete $(k) \Longrightarrow$ $d l f_{k} \wedge \bigvee_{\substack{\alpha \in I_{\alpha}+\\ \alpha \cap A_{k} \neq \emptyset}}\left(G^{\alpha}\right)^{\prime}$.

Definition 12 (Blocking states). For $k \in K$, let $d l f_{k}$ be some non-empty invariant on $\mathbf{X}_{k}$ implying deadlock-freedom of $k$, that is, $d l f_{k} \Rightarrow \bigvee_{a \in A_{k}} G^{a}$. We take $d l f=\bigwedge_{k \in K} d l f_{k}$ and define for a component $k$ the predicate

$$
\operatorname{blocking}(k)=d l f_{k} \wedge \neg \bigvee_{\substack{\alpha \in I C^{+} \\ \alpha \cap A_{k} \neq \emptyset}}\left(G^{\alpha}\right)^{\prime}
$$

characterizing the states where $k$ is blocked due to interaction or restriction with $U$.

Definition 13 (Dependency graph). Consider a system $S=(B, I M) / U$ built from a set $K$ of components. For each component $k$ put each predicate blocking $(k)$ in the form $\bigvee_{i \in I_{k}} c_{i}$ with $c_{i}=\bigwedge_{k^{\prime} \in K} D_{k^{\prime}, i}$ where $D_{k^{\prime}, i}$ is a predicate depending on $X_{k^{\prime}}$. The dependency graph of $S$ is a labelled bipartite graph with two sets of nodes: the components of $K$, and constraint nodes $\left\{c_{i} \mid \exists k \in K . i \in\right.$ $\left.I_{k}\right\}$, where $I_{k}$ is the set of conjunctive terms occurring in blocking $(k)$. For a component node $k$ and a constraint node $c_{i}$,

- there is an edge from $k$ to $c_{i}$ labeled with $D_{k, i}$ if $D_{k, i} \neq$ false; and

- there is an edge from $c_{i}$ to $k$ labeled with $D_{k, i}$ if $i \in I_{k}$.

Notice that constraint nodes represent conditions under which a component is blocked. If $c$ is a constraint node for a component $k$ then it has at least one incoming and one outcoming edge. At some state, $k$ is blocked if all the predicates labelling the incoming edges of $c$ are true.

Let $\gamma$ be a circuit of the dependency graph of a system $(B, I M) / U$. The predicate

$$
D L(\gamma)=\bigwedge_{\left(k \stackrel{D}{\rightarrow} c \stackrel{D^{\prime}}{\rightarrow} k^{\prime}\right) \in \gamma}\left(D \wedge D^{\prime}\right)
$$


characterizes the system states for which all components in $\gamma$ may be blocked cyclically awaiting for each other.

Theorem 1 (Deadlock freedom). A system $(B, I M) / U$ is deadlock-free from any state satisfying $d l f \wedge U^{X}$ if its dependency graph contains a non-empty subgraph $G$ such that

- if $G$ contains a component node then $G$ contains all its predecessor constraint nodes, and if $G$ contains a constraint node then $G$ contains one of its predecessors; and

- for any elementary circuit $\gamma$ of $G, D L(\gamma)=$ false.

Proof. Assume that the system is at some global deadlock state $\mathbf{x}$. As all the components are initialised at states satisfying $d l f$ there exists for each component at least one action $a$ having its guard $G^{a}$ enabled at this state in $B$.

Consider a component $k$ of $G$ and a term $c_{i}$ of blocking $(k)$ such that $c_{i}(\mathbf{x})$. As $d l f_{k}(\mathbf{x})$, the label of the input edge $\left(c_{i}, k\right)$ is true at this state (the guards are contained in $d l f_{k}$ ). Then consider some predecessor $k^{\prime}$ of $c_{i}$ in $G$. The label of the edge $\left(k^{\prime}, c_{i}\right)$ is true as it is a factor of $c_{i}$. Again, the component $k^{\prime}$ has at least one guard enabled. Move backwards in $G$ from this node by iterating the process. Then an elementary circuit $\gamma$ of $G$ is found. By construction, all the predicates labelling the edges of this circuit are true at state $\mathbf{x}$. This contradicts the assumption of $D L(\gamma)=$ false.

Theorem 2 (Deadlock freedom). (B,IM)/U is deadlock-free from $d l f \wedge U^{X}$ if $d l f \wedge U^{X} \Rightarrow \bigvee_{\alpha \in I C^{+}}\left(G^{\alpha}\right)^{\prime}$.

Proof. Since for any $k \in K, d l f_{k}$ is invariant by hypothesis, $d l f$ and $d l f \wedge U^{X}$ are by property 2 invariants of $(B, I M) / U$. As $d l f \wedge U^{X}$ implies enabledness of some complete interaction, its invariance amounts to deadlock-freedom.

Theorem 3. The conditions of theorem 1 imply that $d l f \wedge U^{X} \Rightarrow \bigvee_{\alpha \in I C^{+}}\left(G^{\alpha}\right)^{\prime}$.

Proof (sketch). Suppose that there is some non-empty sub-graph $G$ of the dependency graph as specified in theorem 1 such that for any elementary circuit $\gamma$ of $G, D L(\gamma)=$ false. Thus, for any valuation $\mathbf{x}$ such that $d l f(\mathbf{x})$ there is some component $k$ in $G$ with $\neg$ blocking $(k)(\mathbf{x})$ by construction of the dependency graph. By definition of blocking, it follows that there is some interaction $\alpha$ such that $\left(G^{\alpha}\right)^{\prime}(\mathbf{x})$.

The condition of theorem 2 allows an efficient check for deadlock freedom. However, in order to provide a diagnostic when this condition is not verified, it may be useful to construct a dependency graph for the states satisfying $d l f \wedge$ $\neg \bigvee_{\alpha \in I C^{+}}\left(G^{\alpha}\right)^{\prime}$. 


\subsection{Individual Deadlock-Freedom}

We give some results about deadlock-freedom preservation for transitions systems. Similar results have been obtained for timed transition systems with priorities in [5].

In general, deadlock-freedom or even liveness of a system of interacting components do not imply that any component remains deadlock-free in the system. Guaranteeing this stronger property is the objective of the following definitions and theorem.

Definition 14 (Run). A run of a transition system $B=\left(X, I C,\left\{G^{a}\right\}_{a \in I C}\right.$, $\left.\left\{F^{a}\right\}_{a \in I C}\right)$ is an infinite sequence of interactions $\mathbf{x}_{0} \stackrel{\alpha_{0}}{\rightarrow} \mathbf{x}_{1} \stackrel{\alpha_{1}}{\rightarrow} \ldots \mathbf{x}_{n} \stackrel{\alpha_{n}}{\rightarrow} \ldots$

Definition 15 (Individual deadlock-freedom). Given a system S, a component $k \in K$ is deadlock-free in $S$ if for any run $\sigma$ of $S$ and any prefix $\sigma_{n}$ of $\sigma$, there exists a run $\sigma^{\prime}$ such that $\sigma_{n} \sigma^{\prime}$ is a run of $S$, and some interaction of $\sigma^{\prime}$ contains an interaction of $k$.

Definition 16 (Controllable predecessors). Let $B=\left(X, I C,\left\{G^{a}\right\}_{a \in I C}\right.$, $\left.\left\{F^{a}\right\}_{a \in I C}\right)$ be the behavior of a component. For $Y \subseteq \mathbf{X}$, define pre $(Y) \subseteq \mathbf{X}$ such that $\mathbf{x} \in \operatorname{pre}(Y)$ if

- if $\mathbf{x}$ is complete then $\exists \mathbf{x}^{\prime} \in \mathbf{X} \exists a \in I C^{+} . \mathbf{x} \stackrel{a}{\rightarrow} \mathbf{x}^{\prime} \wedge \mathbf{x}^{\prime} \in Y$;

- if $\mathbf{x}$ is incomplete then $\forall \mathbf{x}^{\prime} \in \mathbf{X} \forall a \in I C^{-} . \mathbf{x} \stackrel{a}{\rightarrow} \mathbf{x}^{\prime} \Rightarrow \mathbf{x}^{\prime} \in Y$, and such $a$ and $\mathbf{x}^{\prime}$ exist.

For $\mathbf{X}_{0} \subseteq \mathbf{X}$ we denote by $\operatorname{PRE}\left(\mathbf{X}_{0}\right)$ the least solution of $Y=\mathbf{X}_{0} \cup \operatorname{pre}(Y)$.

Clearly, $\operatorname{PRE}\left(\mathbf{X}_{0}\right)$ exists, as it is the fixed point of a monotonic functional. $\operatorname{PRE}\left(\mathbf{X}_{0}\right)$ represents the set of the predecessors of $\mathbf{X}_{0}$ in the transition graph such that from anyone of its states a state of $\mathbf{X}_{0}$ can be reached by appropriately choosing complete interactions. In this context, complete interactions can be characterized as controllable, as when they are enabled some interaction containing them can occur in the product system. On the contrary incomplete interactions are uncontrollable as their occurrence in the product depends on the state of the environment. Predicate transformers taking into account controllability have been studied in [13].

Definition 17 (Controllability). Given a system $S$, we call a component $k \in$ $K$ with behavior $\left(X_{k}, A_{k},\left\{G^{a}\right\}_{a \in A_{k}},\left\{F^{a}\right\}_{a \in A_{k}}\right)$ controllable with respect to some state constraint $U$ on $X_{k}$ if $\operatorname{PRE}(U)=X_{k}$.

Theorem 4 (Individual Deadlock-Freedom). Given a system $S=(B, I M)$ built from a set $K$ of components, a component $k \in K$ is deadlock-free from dlf in $S$ if

- the dependency graph of $S$ contains a sub-graph $G$ satisfying the requirements of theorem 1 with $k \in K^{\prime}$, where $K^{\prime}$ is the set of component nodes in $G$, 
- any $k \in K^{\prime}$ is controllable with respect to $G^{a}$ for any a $\in A_{k}$ such that $\exists \alpha \in I C \exists \alpha^{\prime} \in I C^{-} . a \in \alpha \wedge \alpha^{\prime} \subseteq \alpha$, and

- all $n$-ary interactions with $n \geqslant 3$ are rendez-vous, that is, for any interactions $\alpha \in I C^{+}$with $|\alpha| \geqslant 3$ and $\alpha^{\prime} \in I C$, we have (1) if $\alpha \cap \alpha^{\prime} \neq \emptyset$, then $\alpha^{\prime} \in I C^{-}$, and (2) if $a \in \alpha \cap A_{i}$ and $a^{\prime} \in \alpha^{\prime} \cap A_{i}$, then $G^{a} \wedge G^{a^{\prime}}=$ false for any $i \in K$.

Notice that under the hypotheses above, any component that is blocked, is either waiting for one (or more) binary interaction, or for exactly one $n$-ary interaction with $n \geqslant 3$.

Proof. Consider some product state $\mathbf{x}$ in which $k$ is blocked, and no complete interaction involving $k$ is enabled. By theorem 1, some (direct or transitive) predecessor of $k$ can progress. Let $k_{i_{1}} \stackrel{\alpha_{1}}{-\rightarrow} k_{i_{2}} \stackrel{\alpha_{2}}{-\rightarrow} \ldots \stackrel{\alpha_{n-1}=\alpha}{--\rightarrow} k_{i_{n}}=k$ be a chain of components in $K^{\prime}$ where $k_{i} \stackrel{\alpha}{-\rightarrow} k_{j}$ means that $k_{j}$ is in an incomplete state waiting for interaction $\alpha$ with $k_{i}$, and such that only $k_{i_{1}}$ is able to progress. By controllability, $k_{i_{1}}$ can be led (by appropriately choosing some complete action, or by any incomplete action) towards a state $\mathbf{x}^{\prime}$ where its action participating in $\alpha_{1}$ is enabled. If $\alpha_{1}$ is binary then this unblocks $k_{i_{2}}$; otherwise the interaction is by hypothesis a rendez-vous, and both components $k_{i_{1}}$ and $k_{i_{2}}$ remain blocked. In that case we apply the same reasoning to any chain of components blocking $k_{i_{2}}$. Finally, $\alpha_{2}$ will be enabled. The same reasoning can now be applied to $k_{i_{3}}$ and recursively descending the chain, until $\alpha$ becomes enabled.

\section{Discussion}

This work is in progress and needs further validation by examples and case studies. It pursues similar objectives as the work by Th. Henzinger and his colleagues [6]. It lies within the scope of a lasting research program.

The paper presents compositionality and composability results for deadlockfrom of systems built from components following the proposed construction methodology. The concept of component is very general and can be applied to various types of descriptions, e.g. a block of code, hardware, provided they have disjoint state spaces and well defined interface and behavior. Interaction models provide a powerful framework for synchronization, that encompasses both strict and non strict synchronization. Restriction appears to be a very useful concept for imposing global invariants. The layered description principle allows separation of concerns and has been used to some extent in [1]. Furthermore, layered structuring is instrumental for the definition of an associative composition operation.

The provided sufficient deadlock-freedom conditions require that components satisfy specific properties such as existence of non trivial deadlock-free invariants, and of sets of controllable predecessors. These properties can be checked algorithmically only when the components are finite-state. Deadlock-freedom conditions also require the computation of the guards of the constructed system, which may be a source of exponential explosion for general interaction models and restrictions. 


\section{References}

1. K. Altisen, G. Gössler, and J. Sifakis. Scheduler modeling based on the controller synthesis paradigm. Journal of Real-Time Systems, special issue on "controltheoretical approaches to real-time computing", 23(1/2):55-84, 2002.

2. A. Benveniste, P. LeGuernic, and Ch. Jacquemot. Synchronous programming with events and relations: the SIGNAL language and its semantics. Science of Computer Programming, 16:103-149, 1991.

3. J.A. Bergstra and J.W. Klop. Algebra of communicating processes with abstraction. TCS, 37(1):77-121, 1985.

4. G. Berry and G. Gonthier. The ESTEREL synchronous programming language: Design, semantics, implementation. Science of Computer Programming, 19(2):87$152,1992$.

5. S. Bornot, G. Gössler, and J. Sifakis. On the construction of live timed systems. In S. Graf and M. Schwartzbach, editors, Proc. TACAS'00, volume 1785 of LNCS, pages 109-126. Springer-Verlag, 2000.

6. L. de Alfaro and T.A. Henzinger. Interface theories for component-based design. In T.A. Henzinger and C. M. Kirsch, editors, Proc. EMSOFT'01, volume 2211 of LNCS, pages 148-165. Springer-Verlag, 2001.

7. E.W. Dijskstra. A Discipline of Programming. Prentice Hall, 1976.

8. G. Goessler and J. Sifakis. Composition for component-based modeling. In proc. FMCO'02 (to appear), LNCS. Springer-Verlag, 2003.

9. N. Halbwachs, P. Caspi, P. Raymond, and D. Pilaud. The synchronous dataflow programming language LUSTRE. Proceedings of the IEEE, 79(9):1305-1320, September 1991.

10. T.A. Henzinger, B. Horowitz, and C. Meyer Kirsch. Embedded control systems development with Giotto. In Proc. LCTES'01, 2001.

11. C. A. R. Hoare. Communicating Sequential Processes. Prentice Hall, 1985.

12. Hermann Kopetz. Real-Time Systems: Design Principles for Distributed Embedded Applications. Kluwer, 1997.

13. O. Maler, A. Pnueli, and J. Sifakis. On the synthesis of discrete controllers for timed systems. In E.W. Mayr and C. Puech, editors, STACS'95, volume 900 of LNCS, pages 229-242. Springer-Verlag, 1995.

14. F. Maraninchi. Operational and compositional semantics of synchronous automaton compositions. In proc. CONCUR, volume 630 of LNCS. Springer-Verlag, 1992.

15. R. Milner. Calculi for synchrony and asynchrony. Theoretical Computer Science, 25(3):267-310, 1983.

16. J. Misra and K.M. Chandy. Parallel Program Design: A Foundation. AddisonWesley, 1988.

17. K.J. Turner, editor. Using Formal Description Techniques - An Introduction to Estelle, LOTOS and SDL. Wiley, 1993. 